\title{
COMPLETELY BOUNDED POLYNOMIALS BETWEEN OPERATOR SPACES
}

\author{
SEÁN DINEEN and CRISTINA RADU*
}

\begin{abstract}
In this article we introduce the concept of a completely bounded polynomial between operator spaces, prove some basic properties and determine the operator structure of the space of diagonal polynomials on the more important operator Hilbert spaces.
\end{abstract}

\section{Operator Spaces}

Two important areas within functional analysis are Banach space theory and the theory of $\mathscr{C}^{*}$ algebras. In Banach space theory the isomorphic approach has always maintained it's importance while in $\mathscr{C}^{*}$ algebra theory there is only an isometric approach. In the last few decades a new theory, intermediate between the above two, Operator Space Theory, has emerged and come of age in recent years with the publication of books by Effros and Ruan [2], Paulsen [5] and Pisier [6]. This theory of Banach spaces is turned into an essentially isometric theory by building into the definition the isometric realisation of the Banach space as a subset of a $\mathscr{C}^{*}$ algebra.

Guided by the Banach space theory we see that there are a number of different approaches to defining polynomial mappings between operator spaces, e.g. tensor products and duality, multilinear mappings, etc. We use here differentiation, a mapping $P: X \rightarrow Y$ is a polynomial of degree $m$ if and only if for each $v \in X$ the mapping

$$
x \in X \rightarrow D_{v} P(x):=\lim _{t \in \mathrm{C} \rightarrow 0} \frac{P(x+t v)-P(x)}{t} \in Y
$$

is a polynomial of degree $m-1$.

Polynomials of degree greater than 1 are non-linear and the successful linearisation of polynomial mappings between Banach spaces has led to a rich theory of polynomial and holomorphic mappings between Banach spaces [1].

\footnotetext{
* This work was carried out with the partial support of SFI grant R9317. AMS clasification $46 \mathrm{G} 20$.

Received 27 April 2009, in final form 9 October 2009.
} 
In considering polynomials between operator spaces we encounter two sources of non-linearity, that arising from the nature of polynomials, as mentioned above, and in addition the non-linearity due to the matrix norms of operator spaces (see Definition 3 below). This double dose of non-linearity considerably complicated our endeavours and rendered certain proofs, which one might expect from the Banach space theory to be straightforward, non-trivial. The nature of these proofs and the examples given in Section 3, however, have convinced us that the theory of completely bounded (CB) polynomials between operator spaces contains challenging problems and should yield interesting and elegant results.

We now recall the basic concepts from operator space theory. All vector spaces are over the complex numbers and if $n$ is a positive integer we let $M_{n}(V)$ denote the set of all $n \times n$ matrices with entries from the vector space $V$. If $T: V \rightarrow W$ is a linear mapping between vector spaces we let $T_{n}: M_{n}(V) \rightarrow$ $M_{n}(W)$ denote the induced map $T_{n}\left(\left[x_{i j}\right]_{1 \leq i, j \leq n}\right):=\left[T\left(x_{i j}\right)\right]_{1 \leq i, j \leq n}$.

If $X$ and $Y$ are Banach spaces we let $\mathscr{L}(X, Y)$ denote the space of all continuous linear mappings from $X$ to $Y$ and if $T \in \mathscr{L}(X, Y)$ let $\|T\|:=$ $\sup \{\|T(x)\|:\|x\| \leq 1\}$. We let $\mathscr{L}(X, \mathrm{C})=X^{*}, \mathscr{L}(X, X)=\mathscr{B}(X)$ and $\mathscr{H}$ will always denote a Hilbert space. If $\mathscr{C}_{n}$ is an $n$-dimensional Hilbert space then $\mathscr{B}\left(\mathscr{H}_{n}\right)=M_{n}(\mathrm{C})=: M_{n}$ is always assumed to be endowed with the operator norm $\|\cdot\|_{M_{n}}$.

Definition 1.1. An operator space is a triple $(X, i, \mathscr{B}(\mathscr{H}))$ where $X$ is a normed linear space and $i: X \mapsto \mathscr{B}(\mathscr{H})$ is an isometric embedding. If $X$ is isometric as a normed linear space to a Hilbert space we call $(X, i, \mathscr{B}(\mathscr{H}))$ an operator Hilbert space.

It is convenient to sometimes write $X$ in place of $(X, i, \mathscr{B}(\mathscr{H}))$. As a Banach space may be embedded in the space of bounded linear operators on a Hilbert space in many different ways the same Banach space may appear as the Banach space part of many different operator spaces. There are, however, certain natural operator space structures associated with a given operator space and we refer to these as canonical. Unless stated otherwise one may assume that we are using these structures. For example, if $Y$ is a closed subspace of $X, i_{Y}: Y \rightarrow X$ is the canonical embedding, and $(X, i, \mathscr{B}(H))$ is an operator space then we call $\left(Y, i \circ i_{Y}, \mathscr{B}(\mathscr{H})\right)$ the canonical operator space structure on $Y$ inherited from $X$.

If $\mathscr{H}$ is a Hilbert space and $n$ is a positive integer we endow $\mathscr{H}^{n}$ with a Hilbert space structure by letting

$$
\left\langle\left(x_{j}\right)_{j=1}^{n},\left(y_{j}\right)_{j=1}^{n}\right\rangle:=\sum_{j=1}^{n}\left\langle x_{j}, y_{j}\right\rangle
$$


denote the inner product and define a natural embedding

$$
\varphi_{n}: M_{n}(\mathscr{B}(\mathscr{H})) \rightarrow \mathscr{B}\left(\mathscr{H}^{n}\right)
$$

by letting

$$
\left[\varphi_{n}\left(\left[T_{i, j}\right]_{1 \leq i, j \leq n}\right)\right]\left(x_{j}\right)_{j=1}^{n}:=\left[\sum_{j=1}^{n} T_{i, j} x_{j}\right]_{i=1}^{n} .
$$

If $(X, i, \mathscr{B}(\mathscr{H}))$ is a operator space and $n$ is a positive integer we refer to $\left(M_{n}(X), \varphi_{n} \circ i_{n}, \mathscr{B}\left(\mathscr{H}^{n}\right)\right)$ as the canonical operator space structure on $M_{n}(X)$ inherited from $X$. Note that this defines a norm $\|\cdot\|_{M_{n}(X)}$ on $M_{n}(X)$. If $Y$ is an operator subspace of $X$, that is if $Y$ inherits its operator structure from $X$, then the norm and operator structure on $M_{n}(Y)$ are induced by those on $M_{n}(X)$.

Definition 1.2. If $(X, i, \mathscr{B}(\mathscr{H}))$ and $(Y, j, \mathscr{B}(\mathscr{K}))$ are operator spaces then the linear mapping $u: X \rightarrow Y$ is completely bounded if $\|u\|_{\mathrm{cb}}:=$ $\sup _{n}\left\|u_{n}\right\|<\infty$. We let $\mathrm{CB}(X, Y)$, endowed with the norm $\|\cdot\|_{\mathrm{cb}}$, denote the Banach space of all completely bounded mappings from $X$ to $Y$. A linear bijection $u$ between operator spaces is called a complete isometry if $\|u\|_{\mathrm{cb}}=$ $\left\|u^{-1}\right\|_{\mathrm{cb}}=1$.

If $(X, i, \mathscr{B}(\mathscr{H}))$ is an operator space and $i(X)$ generates a commutative $\mathscr{C}^{*}$ subalgebra of $\mathscr{B}(\mathscr{H})$ ) we say that the operator space structure is minimal and write $X_{\min }$ in place of $(X, i, \mathscr{B}(\mathscr{H}))$ (see [2], Proposition 3.3.1 or [6], Proposition 1.10). Given a Banach space $X$ there exists a unique minimal operator space of the form $(X, i, \mathscr{B}(\mathscr{H}))$, it suffices to use the mapping $i: X \rightarrow$ $\mathscr{C}\left(B_{X^{\prime}}, \sigma\left(X^{\prime}, X\right)\right),[i(x)](\varphi)=\varphi(x)$, where $\|i(x)\|=\|x\|=\max \{|\varphi(x)|$ : $\left.\varphi \in X^{\prime},\|\varphi\| \leq 1\right\}$ and $\mathscr{C}$ denotes continuous complex valued functions.

The maximal operator space associated with the Banach space $X$ is characterised as the unique operator space $X_{\max }$ such that for any operator space $Y$ we have isometrically $\mathrm{CB}\left(X_{\max }, Y\right)=\mathscr{B}(X, Y)$ (see [2] p. 48, [5] or [6] p. 71).

Definition 1.3. The pair $\left(V,\left(\|\cdot\|_{n}\right)_{n=1}^{\infty}\right)$, where $V$ is a vector space and for each $n,\left(M_{n}(V),\|\cdot\|_{n}\right)$ is a Banach space, is called an $L^{\infty}$ matrix normed space if the following hold: for all $x \in M_{n}(V), a, b \in M_{n}$

$$
\|a \cdot x \cdot b\| \leq\|a\|_{M_{n}} \cdot\|x\|_{n} \cdot\|b\|_{M_{n}}
$$

for all positive integers we have for all $x \in M_{n}(V), y \in M_{m}(V)$

$$
\|x \oplus y\|_{n+m}=\max \left(\|x\|_{n},\|y\|_{m}\right)
$$

where $x \oplus y$ is the block diagonal element of $M_{n+m}(V)$ with $x$ and $y$ on the diagonal. 
Ruan's theorem [2], [6] establishes a correspondence between operator spaces and $L^{\infty}$-matrix normed spaces.

THeORem 1.4 (Ruan). If $(X, i, \mathscr{B}(\mathscr{H}))$ is an operator space then $(X$, $\left.\left(\|\cdot\|_{M_{n}(X)}\right)_{n=1}^{\infty}\right)$ is an $L^{\infty}$-matrix normed space.

Conversely, if $\left(V,\left(\|\cdot\|_{n}\right)_{n=1}^{\infty}\right)$ is an $L^{\infty}$-matrix normed space then there exists a unique isometric embedding $i$ of $X:=M_{1}(V)$ into $\mathscr{B}(\mathscr{H})$ such that, modulo obvious identifications, for all $n$ the norm induced on $M_{n}(X)$ by the operator space $(X, i, \mathscr{B}(\mathscr{H}))$ coincides with $\|\cdot\|_{n}$.

If $X$ and $Y$ are operator spaces then we identify algebraically $M_{n}(\mathrm{CB}(X, Y))$ and $\mathrm{CB}\left(X, M_{n}(Y)\right)$. It is easy to check that the norms on $\mathrm{CB}\left(X, M_{n}(Y)\right)$ satisfy (1) and (2) and hence, by Ruan's theorem, define the canonical operator space structure on $\mathrm{CB}(X, Y)$. If $Y=\mathrm{C}$ this defines an operator space structure on $X^{*}$. One dimensional spaces admit a unique operator space structure and $X^{*}$ and $\mathrm{CB}(X):=\mathrm{CB}(X, \mathrm{C})$ are isometric as Banach spaces.

Let $X:=(X, i, \mathscr{B}(\mathscr{H}))$ and $Y:=(Y, j, \mathscr{B}(\mathscr{K}))$ denote operator spaces and let $\mathscr{H} \otimes_{2} \mathscr{K}$ denote the Hilbert tensor product of $\mathscr{H}$ and $\mathscr{K}$. We embed $X \otimes Y$ in $\mathscr{B}\left(\mathscr{H} \otimes_{2} \mathscr{K}\right)$ by letting $(x \otimes y)(a \otimes b)=x(a) \otimes y(b)$ and we call the resulting tensor product $X \otimes_{\min } Y$ the minimal operator space tensor product ${ }^{1}$ of $X$ and $Y$. We denote the completed tensor product by $X \bar{\otimes}_{\min } Y$ and denote the norm by $\|\cdot\|_{X \otimes_{\min } Y}$. The norm $\otimes_{\min }$ is a cross norm, that is if $x \in X$ and $y \in Y$ then $\|x \otimes y\|_{X \otimes_{\min } Y}=\|x\| \cdot\|y\|$ and $X \otimes_{\min } M_{n}=M_{n}(X)$ for any operator space $X$ and any positive integer $n$. The minimal tensor product corresponds to the minimal tensor product in $\mathscr{C}^{*}$-algebra theory and to the injective or $\epsilon$ tensor product in Banach space theory. The minimal tensor product is commutative or symmetric, associative and injective, that is it respects subspaces. If $K$ is a compact Hausdorff topological space then

$$
M_{n}(\mathscr{C}(K))=\mathscr{C}\left(K, M_{n}\right)=\mathscr{C}(K) \widehat{\otimes}_{\epsilon} M_{n}=\mathscr{C}(K) \otimes_{\min } M_{n}
$$

as Banach spaces, operator spaces or $\mathscr{C}^{*}$-algebras.

If $X$ is an operator space then, by ([2], p. 139 or [6], p. 40), the natural embedding

$$
\theta_{1, X^{*}, Y}: X^{*} \bar{\otimes}_{\min } Y \rightarrow \mathrm{CB}(X, Y)
$$

given by $\left[\theta_{1, X^{*}, Y}\left(x^{*} \otimes y\right)\right](x)=x^{*}(x) y$, for all $x^{*} \in X^{*}, y \in Y$ and $x \in X$, and linearity is a complete isometric embedding which is surjective if either $X$ or $Y$ is finite dimensional.

\footnotetext{
${ }^{1}$ The notation $\stackrel{\vee}{\otimes}$ and $\|\cdot\|_{\vee}$ are also used in place of $\bar{\otimes}_{\text {min }}$ and $\|\cdot\|$ respectively and $\bar{\otimes}_{\text {min }}$ is also called the injective operator tensor product and the spatial operator tensor product.
} 
Associativity of $\bigotimes_{\min }$ implies that

$$
\bigotimes_{\min }^{m+1} X:=\left(\bigotimes_{\min }^{m} x\right) \bar{\otimes}_{\min } X=\left(\bigotimes_{\min }^{i} x\right) \bar{\otimes}_{\min }\left(\bigotimes^{j} x\right)
$$

for any operator space $X$ whenever $i+j=m+1$.

If $\mathscr{H}$ is a separable ${ }^{2}$ Hilbert space with basis $\left(e_{i}\right)_{i=1}^{\infty}$ then each $T \in \mathscr{B}(\mathscr{H})$ can be identified with the infinite matrix $\left(\left\langle T e_{i}, e_{j}\right\rangle\right)_{1 \leq i, j<\infty}$. The set of all $T \in \mathscr{B}(\mathscr{H})$ with zero entries except in the first row (respectively column) can be identified with $\mathscr{L}(\mathscr{H}, \mathrm{C})$ (respectively $\mathscr{L}(\mathrm{C}, \mathscr{H}))$ and the Hilbert operator spaces $\mathscr{H}_{r}:=R:=(R, i, \mathscr{B}(\mathscr{H}))\left(\right.$ respectively $\mathscr{H}_{c}:=C:=(C, i, \mathscr{B}(\mathscr{H}))$ ), where $i$ is the canonical embedding of $R$ and $C$ into $\mathscr{B}(\mathscr{H})$ are called the row and column operator spaces respectively. We have completely isometrically $R^{*}=C$, and $C^{*}=R$.

Pisier [6] has shown that for any cardinal number $I$ there exists a unique, up to complete isometry, operator Hilbert space $\mathrm{OH}_{I}$ isometric to $l_{2}(I)$ and which is such that the canonical identification $l_{2}(I) \simeq \overline{l_{2}(I)^{*}}$ induces a complete isometry from $\mathrm{OH}_{I}$ to $\overline{\mathrm{OH}_{I}^{*}}$. If cardinality is not an issue we write $\mathrm{OH}$ in place of $\mathrm{OH}_{I}$.

Let $X$ be an operator space and let $\left(e_{i}\right)_{i=1}^{n}$ denote an orthonormal subset of OH. If $\left(x_{i}\right)_{i=1}^{n} \subset X$ then, by [6] p. 146,

$$
\left\|\sum_{i=1}^{n} x_{i} \otimes e_{i}\right\|_{X \bar{\otimes}_{\min } \mathrm{OH}}=\left\|\sum_{i=1}^{n} x_{i} \otimes \bar{x}_{i}\right\|_{X \bar{\otimes}_{\min } X}^{1 / 2} .
$$

Given Hilbert spaces $\mathscr{H}$ and $\mathscr{K}$, we have ([2], p. 163) the complete isometry

$$
\mathscr{H}_{r} \bar{\otimes}_{\min } \mathscr{K}_{r}=\left(\mathscr{H} \otimes_{2} \mathscr{K}\right)_{r} .
$$

\section{Polynomial Mappings between Banach spaces}

If $X$ and $Y$ are Banach spaces and $m$ is a positive integer we let $\mathscr{P}\left({ }^{m} X, Y\right)$ denote the space of continuous $Y$-valued $m$-homogeneous polynomials on $X$. Endowed with the supremum norm over the unit ball of $X,\|\cdot\|$, this space is a Banach space.

If $P \in \mathscr{P}\left({ }^{m} X, Y\right)$ and their exist bounded sequences $\left(\phi_{j}\right)_{j=1}^{\infty} \subset X^{*}$ and $\left(y_{j}\right)_{j=1}^{\infty} \subset Y$ such that $P=\sum_{j=1}^{\infty} \phi_{j}^{m} \cdot y_{j}$ and $\sum_{j=1}^{\infty}\left\|\phi_{j}\right\|^{m} \cdot\left\|y_{j}\right\|<\infty$ then we say that $P$ is nuclear. The nuclear norm on $\mathscr{P}_{N}\left({ }^{m} X, Y\right)$, the space of all

\footnotetext{
${ }^{2}$ We confine ourselves to finite and separable Hilbert spaces purely for convenience.
} 
nuclear $m$-homogeneous polynomials from $X$ to $Y,\|\cdot\|_{N}$, is defined as follows:

$$
\|P\|_{N}:=\inf \left\{\sum_{j=1}^{\infty}\left\|\phi_{j}\right\|^{m} \cdot\left\|y_{j}\right\|: P=\sum_{n=1}^{\infty} \phi_{j}^{m} \cdot y_{j} \cdot\right\} .
$$

Let $\alpha_{m}: \mathscr{P}\left({ }^{m+1} X, Y\right) \rightarrow \mathscr{L}\left(X, \mathscr{P}\left({ }^{m} X, Y\right)\right), \alpha_{m}(P)=\bar{P}$ where

$$
[\bar{P}(x)](y): \stackrel{\vee}{P}(x, y, y, \ldots, y)=\stackrel{\vee}{P}\left(x, y^{m}\right)
$$

and $\stackrel{\vee}{P}: E^{m+1} \rightarrow F$ is the symmetric $(m+1)$-linear map associated with $P$. It is easily verified that

$$
\begin{aligned}
\operatorname{Im}\left(\alpha_{m}\right)=\{T \in \mathscr{L}(X, & \left.\mathscr{P}\left({ }^{m} X, Y\right)\right):\left[T\left(x_{1}\right)\right]^{\vee}\left(x_{2}, \ldots, x_{m+1}\right) \\
& \left.=\left[T\left(x_{\sigma(1)}\right)\right]^{\vee}\left(x_{\sigma(2)}, \ldots, x_{\sigma(m+1)}\right), \text { all } \sigma \in S_{m+1}\right\}
\end{aligned}
$$

where $S_{m+1}$ is the set of all permutations of the first $m+1$ natural numbers.

If $\left(e_{j}\right)_{j=1}^{\infty}$ is a unit vector basis for the Banach space $X$ and $Y$ is a Banach space, then we call $P: X \rightarrow Y$ an $m$-homogeneous diagonal polynomial with respect to $\left(e_{j}\right)_{j=1}^{\infty}$ if there exist a sequence in $Y,\left(y_{j}\right)_{j=1}^{\infty}$ such that

$$
P\left(\sum_{j=1}^{\infty} x_{j} e_{j}\right)=\sum_{j=1}^{\infty} x_{j}^{m} y_{j}
$$

for all $\sum_{j=1}^{\infty} x_{j} e_{j} \in X$ and

$$
\lim _{n \rightarrow \infty} \sup \left\{\left\|P\left(\sum_{j=n}^{\infty} x_{j} e_{j}\right)\right\|:\left\|\sum_{j=1}^{\infty} x_{j} e_{j}\right\| \leq 1\right\} \rightarrow 0
$$

as $n \rightarrow \infty$. This means in particular that $\left\|y_{j}\right\|=\left\|P\left(e_{j}\right)\right\| \rightarrow 0$ as $j \rightarrow$ $\infty$. As the basis is generally fixed we use the term diagonal polynomial and let $\mathscr{P}_{d}\left({ }^{m} X, Y\right)$ denote the space of all continuous $m$-homogenous diagonal polynomials from $X$ to $Y$.

\section{Completely Bounded Polynomials}

We now define inductively the completely bounded polynomials between operator spaces and show they can be endowed naturally with an operator space structure. If $X$ and $Y$ are operator spaces let

$$
\mathscr{P}_{\mathrm{cb}}\left({ }^{1} X, Y\right)=\mathrm{CB}(X, Y)
$$


as operator spaces. Now suppose that we have defined, as an operator space, $\mathscr{P}_{\mathrm{cb}}\left({ }^{m} X, Y\right)$ where $m$ is a positive integer. This implies that $\mathrm{CB}\left(X, \mathscr{P}_{\mathrm{cb}}\left({ }^{m} X, Y\right)\right)$ also has a canonical operator space structure and allows us to make the following definition.

Definition 3.1. Let

$$
\mathscr{P}_{\mathrm{cb}}\left({ }^{m+1} X, Y\right):=\left\{P \in \mathscr{P}\left({ }^{m+1} X, Y\right): \bar{P} \in \mathrm{CB}\left(X, \mathscr{P}_{\mathrm{cb}}\left({ }^{m} X, Y\right)\right)\right\} .
$$

In light of earlier remarks we note that

$$
[m \bar{P}(y)](x)=m \stackrel{\vee}{P}\left(x^{m-1}, y\right)=D_{y} P(x)
$$

for all $x, y \in X$. We let $\|\cdot\|_{\bullet}$ denote the norm on $\mathscr{P}_{\mathrm{cb}}\left({ }^{m} X, Y\right)$ and let $\|\cdot\|_{\mathrm{cb}}$ denote the norm on $\mathrm{CB}\left(X, \mathscr{P}_{\mathrm{cb}}\left({ }^{m} X, Y\right)\right)$, that is $\|\cdot\|_{\mathrm{cb}}$ is the completely bounded norm of completely bounded linear mappings from $X$ into $\mathscr{P}_{\mathrm{cb}}\left({ }^{m} X, Y\right)$.

Lemma 3.2. If $X$ and $Y$ are operator spaces and $m$ and $n$ are positive integers then $M_{n}\left(\mathscr{P}_{\mathrm{cb}}\left({ }^{m} X, Y\right)\right)=\mathscr{P}_{\mathrm{cb}}\left({ }^{m} X, M_{n}(Y)\right)$ as operator spaces.

Proof. Since we may identify $M_{n}(\mathrm{CB}(X, Y))$ and $\mathrm{CB}\left(X, M_{n}(Y)\right)$ for any operator spaces $X$ and $Y$ (see [2], p. 45) the result is true when $m=1$. Suppose the result holds for $m$. By induction, the $m=1$ case and injectivity of $\otimes_{\min }$ we obtain the sequence of complete isometric embeddings:

$$
\begin{aligned}
M_{n}\left(\mathscr{P}_{\mathrm{cb}}\left({ }^{m+1} X, Y\right)\right) & =M_{n} \otimes_{\min } \mathscr{P}_{\mathrm{cb}}\left({ }^{m+1} X, Y\right) \\
& \rightarrow M_{n} \otimes_{\min } \mathrm{CB}\left(X, \mathscr{P}_{\mathrm{cb}}\left({ }^{m} X, Y\right)\right) \\
& =M_{n}\left(\mathrm{CB}\left(X, \mathscr{P}_{\mathrm{cb}}\left({ }^{m} X, Y\right)\right)\right) \\
& =\mathrm{CB}\left(X, M_{n}\left(\mathscr{P}_{\mathrm{cb}}\left({ }^{m} X, Y\right)\right)\right) \\
& =\mathrm{CB}\left(X, \mathscr{P}_{\mathrm{cb}}\left({ }^{m} X, M_{n}(Y)\right)\right) .
\end{aligned}
$$

The characterisation of $\alpha_{m}$ in the previous section shows that the image of $M_{n}\left(\mathscr{P}_{\mathrm{cb}}\left({ }^{m+1} X, Y\right)\right)$ coincides with $\mathscr{P}_{\mathrm{cb}}\left({ }^{m+1} X, M_{n}(Y)\right)$. This completes the proof.

Let $\|\cdot\|_{M_{n}^{m}(X, Y)}$ denote the norm on $\mathscr{L}\left(M_{n}(X), \mathscr{P}_{\mathrm{cb}}\left({ }^{m} X, M_{n}(Y)\right)\right)$ for positive integers $m$ and $n$. The above identifications mean that we have

$$
\|P\|_{\bullet}=\|\bar{P}\|_{\mathrm{cb}}=\sup _{n}\left\|\bar{P}_{n}\right\|_{M_{n}^{m}(X, Y)}
$$

for all $P \in \mathscr{P}_{\mathrm{cb}}\left({ }^{m+1} X, Y\right)$. 
Proposition 3.3. Let $X, Y$ be operator spaces and suppose $Y$ is complete. For every positive integer $m,\left(\mathscr{P}_{\mathrm{cb}}\left({ }^{m} X, Y\right),\|\cdot\|_{\bullet}\right)$ is a Banach space and $\|P\| \leq$ $\|P\|_{\bullet}$ for all $P \in \mathscr{P}_{\mathrm{cb}}\left({ }^{m} X, Y\right)$, that is the natural embedding

$$
P \in \mathscr{P}_{\mathrm{cb}}\left({ }^{m} X, Y\right) \mapsto P \in \mathscr{P}\left({ }^{m} X, Y\right)
$$

is a continuous contraction.

Proof. By construction $\mathscr{P}_{\mathrm{cb}}\left({ }^{m} X, Y\right) \subset \mathscr{P}\left({ }^{m} X, Y\right)$ for all $m$. We prove the remaining results by induction. The case $m=1$ follows from Definition 2 since $\mathrm{CB}(X, Y)$ is complete.

We suppose the result is true for $m$. If $P \in \mathscr{P}_{\mathrm{cb}}\left({ }^{m+1} X, Y\right)$ then

$$
\bar{P} \in \mathrm{CB}\left(X, \mathscr{P}_{\mathrm{cb}}\left({ }^{m} X, Y\right)\right) \subset \mathscr{L}\left(X, \mathscr{P}_{\mathrm{cb}}\left({ }^{m} X, Y\right)\right)
$$

and using the induction hypothesis

$$
\begin{aligned}
\|P\|_{\bullet}=\|\bar{P}\|_{\mathrm{cb}} & \geq\|\bar{P}\|_{\mathscr{L}\left(X, \mathscr{P}_{\mathrm{cb}}\left({ }^{m} X, Y\right)\right)} \\
& =\sup _{x \in X,\|x\| \leq 1}\|\bar{P}(x)\|_{\mathscr{P}_{\mathrm{cb}}\left({ }^{m} X, Y\right)} \\
& \geq \sup _{x \in X,\|x\| \leq 1}\|\bar{P}(x)\| \mathscr{P}^{\left({ }^{m} X, Y\right)} \\
& =\sup _{x, y \in X,\|x\| \leq 1,\|y\| \leq 1}\left\|\stackrel{\vee}{P}\left(x, y^{m}\right)\right\| \\
& \geq \sup _{x \in X,\|x\| \leq 1}\left\|\stackrel{\vee}{P}\left(x, x^{m}\right)\right\|=\|P\| .
\end{aligned}
$$

Since $\mathscr{P}_{\mathrm{cb}}\left({ }^{m} X, Y\right)$ is complete by our induction hypothesis, it follows that $\mathrm{CB}\left(E, \mathscr{P}_{\mathrm{cb}}\left({ }^{m} X, Y\right)\right)$ is also complete. By (5) and the polarisation formula ([1],p. 9)

$$
\left\{\bar{P}: P \in \mathscr{P}_{\mathrm{cb}}\left({ }^{m+1} X, Y\right)\right\}
$$

is a closed subspace of $\mathrm{CB}\left(E, \mathscr{P}_{\mathrm{cb}}\left({ }^{m} X, Y\right)\right)$. Hence $\left(\mathscr{P}_{\mathrm{cb}}\left({ }^{m+1} X, Y\right),\|\cdot\|_{\bullet}\right)$ is complete. This completes the proof.

Using the fact that

$$
\|\varphi\|=\|\varphi\|_{\mathrm{cb}}=\|\varphi\|_{\bullet}
$$

for all $\varphi \in X^{*}$ we see that if $P:=\varphi^{m+1} \cdot y \in \mathscr{P}\left({ }^{m+1} X, Y\right)$ where $\varphi \in X^{*}, y \in Y$ and $m$ is a positive integer then $\bar{P}(x)=\varphi(x) \varphi^{m} \cdot y$. Hence $P \in \mathscr{P}_{\mathrm{cb}}\left({ }^{m+1} X, Y\right)$ and

$$
\|P\|_{\bullet}=\|\varphi\|^{m+1} \cdot\|y\|=\|P\|=\|P\|_{N} .
$$


This immediately leads to the following proposition which shows that there always exists an abundance of CB-polynomials. For tensors of degree $m$ we let $\bigotimes_{m} x=x \otimes \cdots \otimes x$ ( $m$-times) and let $\bigotimes_{\min }^{s, m} X^{*}$ denote the set of all symmetric tensors in $\bigotimes_{\min }^{m} X^{*}$ with the operator space structure inherited from $\bigotimes_{\min }^{m} X^{*}$.

Proposition 3.4. Let $X$ and $Y$ be operator spaces and let $m$ denote a positive integer.

(a) If $\left(\varphi_{j}\right)_{j=1}^{n} \subset X^{*}$ and $\left(y_{j}\right)_{j=1}^{n} \subset Y$ then $\sum_{j=1}^{n} \varphi_{j}^{m} \cdot y_{j} \in \mathscr{P}_{\mathrm{cb}}\left({ }^{m} X, Y\right)$ and

$$
\left\|\sum_{j=1}^{n} \varphi_{j}^{m} \cdot y_{j}\right\|_{\bullet}=\left\|\sum_{j=1}^{n}\left(\bigotimes_{m} \varphi_{j}\right) \otimes y_{j}\right\|_{\left(\otimes_{\min }^{m} X^{*}\right) \otimes_{\min } Y}
$$

(b) $\mathscr{P}_{N}\left({ }^{m} X\right) \subset \mathscr{P}_{\mathrm{cb}}\left({ }^{m} X\right) \subset \mathscr{P}\left({ }^{m} X\right)$ for all $m$ and

$$
\|P\| \leq\|P\|_{\bullet} \leq\|P\|_{N}
$$

for all $P \in \mathscr{P}_{N}\left({ }^{m} X\right)$.

Proof. (a) Suppose the mapping

$$
\theta_{m, X^{*}, Y}:\left(\bigotimes_{\min }^{s, m} X^{*}\right) \otimes_{\min } Y \rightarrow \mathscr{P}\left({ }^{m} X, Y\right)
$$

defined by linearity and by letting $\theta_{m, X^{*}, Y}\left(\left(\bigotimes_{m} \varphi\right) \otimes y\right)=\varphi^{m} \cdot y$ is a complete isometric embedding. We have already noted in (3) that this is the case when $m=1$. This implies that the mapping

$$
\beta_{m}: T \in \mathrm{CB}\left(X,\left(\bigotimes_{\min }^{s, m} X^{*}\right) \otimes_{\min } Y\right) \rightarrow \theta_{m, X^{*}, Y} \circ T \in \mathrm{CB}\left(X, \mathscr{P}\left({ }^{m} X, Y\right)\right)
$$

is also a complete isometric embedding. Using the mappings $\alpha_{m}$ defined earlier we see that

$$
\alpha_{m} \circ \theta_{m+1, X^{*}, Y}=\beta_{m} \circ \theta_{1, X^{*},\left(\bigotimes_{\min }^{s, m} X^{*}\right) \otimes_{\min } Y} .
$$

The mappings on the right in (8) are complete isometric embeddings and as $\alpha_{m}$ is also a complete isometric embedding it follows that $\theta_{m+1, X^{*}, Y}$ is a complete isometric embedding. This proves (a) by induction. Part (b) follows from (7).

When the domain is a maximal operator space all continuous polynomials are completely bounded. This shows that the theory of completely bounded polynomials includes the theory of continuous polynomials. This is part of the following proposition. 
Proposition 3.5. If $X$ is a maximal operator space, $Y$ is an operator space and $m$ is a positive integer then

$$
\mathscr{P}\left({ }^{m} X, Y\right)=\mathscr{P}_{\mathrm{cb}}\left({ }^{m} X, Y\right)
$$

and

$$
\|P\| \leq\|P\| \bullet \leq \frac{m^{m}}{m !}\|P\|
$$

for all $P \in \mathscr{P}\left({ }^{m} X, Y\right)$.

Proof. When $m=1$ the result holds by the definition of maximal operator spaces. We suppose that the result holds for the positive integer $m$. Let $P \in$ $\mathscr{P}\left({ }^{m+1} X, Y\right)$. Then, by the induction hypothesis, and the definition of maximal operator space

$$
\bar{P} \in \mathscr{L}\left(X, \mathscr{P}\left({ }^{m} X, Y\right)\right)=\mathscr{L}\left(X, \mathscr{P}_{\mathrm{cb}}\left({ }^{m} X, Y\right)\right)=\mathrm{CB}\left(X, \mathscr{P}_{\mathrm{cb}}\left({ }^{m} X, Y\right)\right)
$$

and $P \in \mathscr{P}_{\mathrm{cb}}\left({ }^{m+1} X, Y\right)$. Moreover, for all $P \in \mathscr{P}\left({ }^{m+1} X, Y\right)$ we have

$$
\begin{aligned}
\|P\| \leq\|P\|_{\bullet} & =\|\bar{P}\|_{\mathrm{CB}\left(X, \mathscr{P}\left({ }^{m} X, Y\right)\right)}=\|\bar{P}\|_{\mathscr{L}\left(X, \mathscr{P}_{\mathrm{cb}}\left({ }^{m} X, Y\right)\right)} \quad \text { (since } X \text { is maximal) } \\
& =\sup \{\|\bar{P}(x)\| \bullet:\|x\| \leq 1\} \\
& \leq \frac{m^{m}}{m !} \sup \{\|\bar{P}(x)\|:\|x\| \leq 1\} \quad \text { (by induction) } \\
& =\frac{m^{m}}{m !} \sup \left\{\left\|\frac{1}{m+1} \cdot D_{y} P(x)\right\|:\|x\|,\|y\| \leq 1\right\} \\
& \leq \frac{m^{m}}{m !} \cdot \frac{1}{m+1} \cdot \frac{(m+1)^{m+1}}{m^{m}}\|P\| \quad \text { (by Corollary } 1 \text { in [3]) } \\
& =\frac{(m+1)^{m+1}}{(m+1) !}\|P\| .
\end{aligned}
$$

This completes the proof.

Clearly the left-hand inequality in Proposition 3.5 is always best possible and we now show that the same holds for the right-hand inequality. Let $X=\ell_{1}$ endowed with its maximal operator space structure. If $m$ is positive integer we let $P_{m}\left(\left(x_{n}\right)_{n=1}^{\infty}\right)=x_{1} \ldots x_{m}$ for all $\left(x_{n}\right)_{n=1}^{\infty} \in \ell_{1}$. Clearly $P_{m} \in \mathscr{P}\left({ }^{m} \ell_{1}\right)$ and, by Lemma 1.38 in [1], $\left\|P_{m}\right\|=m^{-m}$ and hence, by Proposition 3.5,

$$
\left\|P_{m}\right\|_{\bullet} \leq \frac{m^{m}}{m !} \cdot \frac{1}{m^{m}}=\frac{1}{m !} .
$$


To show that $\left\|P_{m}\right\|_{\bullet}=\frac{m^{m}}{m !}\left\|P_{m}\right\|$ it suffices to show that $\left\|P_{m}\right\|_{\bullet} \geq \frac{1}{m !}$. This holds when $m=1$ by (7). Suppose the result is true for the positive integer $m$. It is easily checked that

$$
\left[\overline{P_{m+1}}\left(\left(x_{n}\right)_{n=1}^{\infty}\right)\right]\left(\left(y_{n}\right)_{n=1}^{\infty}\right)^{m}=\frac{1}{m+1} \sum_{i=1}^{m+1} y_{1} \ldots y_{i-1} x_{i} y_{i+1} \ldots y_{m+1} .
$$

If $\mathbf{e}=(0, \ldots, 0,1,0, \ldots)$, where 1 lies in the $(m+1)^{\text {th }}$ position, then $\|\mathbf{e}\|=1$ and $\overline{P_{m+1}}(\mathbf{e})=\frac{1}{m+1} P_{m}$, and by induction we have, as required,

$$
\left\|P_{m+1}\right\|_{\bullet} \geq\left\|\overline{P_{m+1}}(\mathbf{e})\right\|_{\bullet}=\frac{1}{m+1}\left\|P_{m}\right\|_{\bullet}=\frac{1}{m+1} \cdot \frac{1}{m !}=\frac{1}{(m+1) !}
$$

We now show that the scalar-valued CB polynomials form an algebra and afterwards consider the composition of certain CB polynomials. The analogous results for continuous polynomials are easily proved and illustrates the difference between continuous and CB polynomials.

Proposition 3.6. Let $X$ denote an operator space and let $m, k$ be natural numbers. If $P \in \mathscr{P}_{\mathrm{cb}}\left({ }^{m} X\right)$ and $Q \in \mathscr{P}_{\mathrm{cb}}\left({ }^{k} X\right)$ then $P Q \in \mathscr{P}_{\mathrm{cb}}\left({ }^{m+k} X\right)$ and

$$
\|P Q\|_{\bullet} \leq\|P\| \bullet Q \|_{\bullet} .
$$

PRoOF. The product rule for differentiation implies for all $x, y \in X$ that

$$
D(P Q)_{y}(x)=D P_{y}(x) Q(x)+P(x) D Q_{y}(x) .
$$

Hence

$$
[(m+k) \overline{P Q}(y)](x)=[m \bar{P}(y)](x) Q(x)+P(x)[k \bar{Q}(y)](x)
$$

that is

$$
(m+k) \overline{P Q}(y)=m \bar{P}(y) Q+k \bar{Q}(y) P .
$$

For any natural number $n$, let

$$
\bar{P}_{n}: M_{n}(X) \rightarrow M_{n}\left(\mathscr{P}_{\mathrm{cb}}\left({ }^{m-1} X\right)\right)=\mathscr{P}_{\mathrm{cb}}\left({ }^{m-1} X, M_{n}\right)
$$

where

$$
m \bar{P}_{n}\left(\left[y_{i j}\right]_{i, j=1}^{n}\right)=m\left[\bar{P}\left(y_{i j}\right)\right]_{i, j=1}^{n}=\left[D_{y_{i j}} P\right]_{i, j=1}^{n} .
$$

Since

$$
\bar{Q}_{n}: M_{n}(X) \rightarrow M_{n}\left(\mathscr{P}_{\mathrm{cb}}\left({ }^{k-1} X\right)\right)=\mathscr{P}_{\mathrm{cb}}\left({ }^{k-1} X, M_{n}\right)
$$


is given by

$$
k \bar{Q}_{n}\left(\left[y_{i j}\right]_{i, j=1}^{n}\right)=k\left[\bar{Q}\left(y_{i j}\right)\right]_{i, j=1}^{n}=\left[D_{y_{i j}} Q\right]_{i, j=1}^{n} .
$$

we have $(\overline{P Q})_{n}: M_{n}(X) \rightarrow \mathscr{P}_{\mathrm{cb}}\left({ }^{m+k-1} X, M_{n}\right)$ and

$$
\begin{aligned}
(m+k)(\overline{P Q})_{n}\left(\left[y_{i j}\right]_{i, j=1}^{n}\right) & =\left[(m+k) \overline{P Q}\left(y_{i j}\right)\right]_{i, j=1}^{n} \\
& =\left[m \bar{P}\left(y_{i j}\right) Q+k \bar{Q}\left(y_{i j}\right) P\right]_{i, j=1}^{n} \\
& =m \bar{P}_{n}\left(\left[y_{i j}\right]_{i, j=1}^{n}\right) Q+k \bar{Q}_{n}\left(\left[y_{i j}\right]_{i, j=1}^{n}\right) P .
\end{aligned}
$$

Since $\overline{P_{n}}\left(\left[y_{i j}\right]_{i, j=1}^{n}\right) \in \mathscr{P}\left({ }^{m-1} X, M_{n}\right)$ and $Q \in \mathscr{P}\left({ }^{k-1} X\right)$ we have

$$
\left\|\overline{P_{n}}\left(\left[y_{i j}\right]_{i, j=1}^{n}\right) Q\right\| \leq\left\|\overline{P_{n}}\left(\left[y_{i j}\right]_{i, j=1}^{n}\right)\right\|\|Q\| \leq\left\|\overline{P_{n}}\left(\left[y_{i j}\right]_{i, j=1}^{n}\right)\right\|\|Q\| . .
$$

Similarly, since $\overline{Q_{n}}\left(\left[y_{i j}\right]_{i, j=1}^{n}\right) \in \mathscr{P}\left({ }^{k-1} X, M_{n}\right)$ and $P \in \mathscr{P}\left({ }^{m-1} X\right)$, we get

$$
\left\|\overline{Q_{n}}\left(\left[y_{i j}\right]_{i, j=1}^{n}\right) P\right\| \leq\left\|\overline{Q_{n}}\left(\left[y_{i j}\right]_{i, j=1}^{n}\right)\right\|\|P\| \leq\left\|\overline{Q_{n}}\left(\left[y_{i j}\right]_{i, j=1}^{n}\right)\right\|\|P\|_{\bullet} .
$$

Hence

$$
\begin{aligned}
(m+k) \sup _{\left\|\left[y_{i j}\right]_{i, j=1}^{n}\right\| \leq 1}\left\|\overline{(P Q)}_{n}\right\| \leq m \sup _{\left\|\left[y_{i j}\right]_{i, j=1}^{n}\right\| \leq 1}\left\|\bar{P}_{n}\left(\left[y_{i j}\right]\right)\right\|\|Q\| . & \\
& +k \sup _{\left\|\left[y_{i j}\right]_{i, j=1}^{n}\right\| \leq 1}\left\|\bar{Q}_{n}\left(\left[y_{i j}\right]\right)\right\|\|P\| \bullet
\end{aligned}
$$

or

$$
(m+k)\left\|\overline{(P Q)}_{n}\right\| \leq m\left\|\bar{P}_{n}\right\|\|Q\|_{\bullet}+k\|P\| \bullet\left\|\bar{Q}_{n}\right\| .
$$

Taking the supremum over the natural numbers $n$ we obtain

$$
(m+k)\|\overline{P Q}\|_{\mathrm{cb}} \leq m\|\bar{P}\|_{\mathrm{cb}}\|Q\|_{\bullet}+k\|\bar{Q}\|_{\mathrm{cb}}\|P\|_{\bullet}
$$

that is

$$
(m+k)\|P Q\|_{\bullet} \leq m\|P\|_{\bullet}\|Q\|_{\bullet}+k\|Q\|_{\bullet}\|P\|_{\bullet}
$$

or

$$
(m+k)\|P Q\|_{\bullet} \leq(m+k)\|P\|_{\bullet}\|Q\| \bullet
$$

and the proof is complete.

Proposition 3.7. Let $X, Y, Z$ and $W$ denote operator spaces, $u: X \rightarrow Y$ and $v: Z \rightarrow W$ cb linear maps and let $P \in \mathscr{P}_{\mathrm{cb}}\left({ }^{m} Y, Z\right)$. Then $v \circ P \circ u \in$ $\mathscr{P}_{\mathrm{cb}}\left({ }^{m} X, W\right)$ and

$$
\|v \circ P \circ u\|_{\bullet} \leq\|v\|_{\mathrm{cb}} \cdot\|P\|_{\bullet} \cdot\|u\|_{\mathrm{cb}}^{m} .
$$


Proof. We use induction on $m$. If $m=1$ then $P \in \mathrm{CB}(Y, Z)$. In this case it is well known, see [2], [5], [6], that $v \circ P \circ u \in \mathrm{CB}(X, W)$ and

$$
\|v \circ P \circ u\|_{\mathrm{cb}} \leq\|v\|_{\mathrm{cb}} \cdot\|P\|_{\mathrm{cb}} \cdot\|u\|_{\mathrm{cb}} .
$$

In the first case we suppose that $v \circ P \in \mathscr{P}_{\mathrm{cb}}\left({ }^{m} Y, W\right)$ and $\|v \circ P\| \bullet$ $\|v\|_{\mathrm{cb}}\|P\|_{\bullet}$ whenever $P \in \mathscr{P}_{\mathrm{cb}}\left({ }^{m} Y, Z\right)$ and $v \in \mathrm{CB}(Z, W)$.

Suppose $P \in \mathscr{P}_{\mathrm{cb}}\left({ }^{m+1} Y, Z\right)$. By the polarisation formula the symmetric $(m+1)$-linear mappings $(v \circ P)^{\vee}$ and $v \circ \stackrel{\vee}{P}$ are equal since they coincide on the diagonal in $Y^{m+1}$. Hence $\overline{v \circ P}=v \circ \bar{P}$ and, by the induction hypothesis, $v \circ \bar{P}(x) \in \mathscr{P}_{\mathrm{cb}}\left({ }^{m} Y, W\right)$ for all $x \in X$. Hence

$$
\overline{v \circ P}: x \in Y \rightarrow \mathscr{P}_{\mathrm{cb}}\left({ }^{m} Y, W\right)
$$

Moreover, if $\left[y_{i j}\right]_{1 \leq i, j \leq n} \in M_{n}(Y)$ then

$$
\begin{aligned}
(\overline{v \circ P})_{n}\left(\left[y_{i j}\right]_{1 \leq i, j \leq n}\right) & =\left[\overline{v \circ P}\left(y_{i j}\right)\right]_{1 \leq i, j \leq n}=\left[v \circ \bar{P}\left(y_{i j}\right)\right]_{1 \leq i, j \leq n} \\
& =v_{n} \circ\left[\bar{P}\left(y_{i j}\right)\right]_{1 \leq i, j \leq n}=v_{n} \circ \bar{P}_{n}\left(\left[y_{i j}\right]_{1 \leq i, j \leq n}\right) .
\end{aligned}
$$

Hence $(\overline{v \circ P})_{n}=v_{n} \circ \bar{P}_{n}$ and

$$
\left\|(\overline{v \circ P})_{n}\right\|_{M_{n}^{m}(Y, W)} \leq\left\|v_{n}\right\|{\mathscr{L}\left(M_{n}(Z), M_{n}(W)\right)}\left\|\bar{P}_{n}\right\|_{M_{n}^{m}(Y, Z)} .
$$

Since this holds for all $n$,

$$
\|v \circ P\|_{\bullet}=\sup _{n}\left\|(\overline{v \circ P})_{n}\right\| \leq \sup _{n}\left\|v_{n}\right\| \cdot \sup _{n}\left\|\bar{P}_{n}\right\|_{M_{n}^{m}(Y, Z)}=\|v\|_{\mathrm{cb}} \cdot\|\bar{P}\|_{\mathrm{cb}}
$$

and

$$
\|v \circ P\|_{\bullet} \leq\|v\|_{\mathrm{cb}} \cdot\|P\|_{\bullet} .
$$

For fixed $u \in \mathscr{L}(X, Y)$ and $P \in \mathscr{P}\left({ }^{m} Y, Z\right)$ consider the sequence of mappings

$$
X \stackrel{u}{\longrightarrow} Y \stackrel{\bar{P}}{\longrightarrow} \mathscr{P}\left({ }^{m} Y, Z\right) \stackrel{\gamma_{m, Z}}{\longrightarrow} \mathscr{P}\left({ }^{m} X, Z\right)
$$

where $\gamma_{m, Z}(Q)=Q \circ u$ for all $Q \in \mathscr{P}\left({ }^{m} Y, Z\right)$.

Our induction hypothesis on $m$ is that the following three conditions hold for all operator spaces $X, Y$ and $Z$, all $u \in \mathrm{CB}(X, Y)$ and all $P \in \mathscr{P}_{\mathrm{cb}}\left({ }^{m} Y, Z\right)$ :

(a) $\gamma_{m, Z}\left(\mathscr{P}_{\mathrm{cb}}\left({ }^{m} Y, Z\right)\right) \subset \mathscr{P}_{\mathrm{cb}}\left({ }^{m} X, Z\right)$,

(b) $\gamma_{m, Z}$ is completely bounded and $\left\|\gamma_{m, Z}\right\|_{\mathrm{cb}} \leq\|u\|_{\mathrm{cb}}^{m}$,

(c) $\|P \circ u\|_{\bullet} \leq\|P\|_{\bullet} \cdot\|u\|_{\mathrm{cb}}^{m}$ for all $P \in \mathscr{P}_{\mathrm{cb}}\left({ }^{m} Y, Z\right)$. 
We first verify the above when $m=1$. If $v \in \mathscr{P}\left({ }^{1} Y, Z\right)=\mathrm{CB}(Y, Z)$ then $\gamma_{1, Z}(v)=v \circ u \in \mathrm{CB}(X, Z)$ and as $\|v \circ u\|_{\mathrm{cb}} \leq\|v\|_{\mathrm{cb}} \cdot\|u\|_{\mathrm{cb}}$ we have

$$
\left\|\gamma_{1, Z}\right\|=\sup \left\{\|v \circ u\|_{\mathrm{cb}}:\|v\|_{\mathrm{cb}} \leq 1\right\} \leq\|u\|_{\mathrm{cb}} .
$$

Since $\left(\gamma_{1, Z}\right)_{n}: \mathrm{CB}\left(Y, M_{n}(Z)\right) \rightarrow \mathrm{CB}\left(X, M_{n}(Z)\right)$ is given by $\left(\gamma_{1, Z}\right)_{n}=$ $\gamma_{1, M_{n}(Z)},(10)$ implies that $\left\|\left(\gamma_{1, Z}\right)_{n}\right\| \leq\|u\|_{\mathrm{cb}}$. Hence

$$
\left\|\gamma_{1, Z}\right\|_{\mathrm{cb}}=\sup _{n}\left\|\left(\gamma_{1, Z}\right)_{n}\right\| \leq\|u\|_{\mathrm{cb}}
$$

and this proves the $m=1$ induction step. Now suppose the inductive hypothesis holds for the positive integer $m$. Take a fixed $P$ in $\mathscr{P}_{\mathrm{cb}}\left({ }^{m+1} Y, Z\right)$. Then $P \circ u \in \mathscr{P}\left({ }^{m+1} X, Z\right)$ and for all $x, y \in X$

$$
\begin{aligned}
{[\overline{P \circ u}(x)](y) } & =(P \circ u)^{\vee}\left(x, y^{m}\right)=\stackrel{\vee}{P}\left(u(x), u(y)^{m}\right) \\
& =[\bar{P}(u(x))](u(y))=([\bar{P}(u(x))] \circ u)(y) \\
& =\left[\gamma_{m, Z}(\bar{P}(u(x)))\right](y) .
\end{aligned}
$$

Hence

$$
\overline{P \circ u}(x)=[\bar{P}(u(x))] \circ u=\gamma_{m, Z}(\bar{P}(u(x)))
$$

and

$$
\overline{P \circ u}=\gamma_{m, Z} \circ \bar{P} \circ u .
$$

As all the terms in the right-hand side of (11) are completely bounded, $\overline{P \circ u}$ is completely bounded, $P \circ u=\gamma_{m+1, Z}(P) \in \mathscr{P}_{\mathrm{cb}}\left({ }^{m+1} X, Z\right)$, and this establishes (a) for $m+1$. By the inductive hypothesis (b),

$$
\|\overline{P \circ u}\|_{\mathrm{cb}} \leq\left\|\gamma_{m, Z}\right\|_{\mathrm{cb}} \cdot\|\bar{P}\|_{\mathrm{cb}} \cdot\|u\|_{\mathrm{cb}} \leq\|u\|_{\mathrm{cb}}^{m} \cdot\|\bar{P}\|_{\mathrm{cb}} \cdot\|u\|_{\mathrm{cb}}
$$

and hence

$$
\|P \circ u\|_{\bullet} \leq\|P\|_{\bullet} \cdot\|u\|_{\mathrm{cb}}^{m+1} .
$$

This proves (c) for $m+1$ and all $Z$. Moreover, as

$$
\|\overline{P \circ u}\|_{\mathrm{cb}}=\|P \circ u\|_{\bullet}=\left\|\gamma_{m+1, Z}(P)\right\|_{\bullet}
$$

(12) also implies

$$
\left\|\gamma_{m+1, Z}\right\| \leq\|u\|_{\mathrm{cb}}^{m+1}
$$


for any operator space $Z$. Now

$$
\left(\gamma_{m+1, Z}\right)_{n}=\gamma_{m+1, M_{n}(Z)}: \mathscr{P}_{\mathrm{cb}}\left({ }^{m+1} Y, M_{n}(Z)\right) \rightarrow \mathscr{P}_{\mathrm{cb}}\left({ }^{m+1} X, M_{n}(Z)\right)
$$

and, replacing $Z$ by $M_{n}(Z)$ in (13), we see that

$$
\left\|\gamma_{m+1, Z}\right\|_{\mathrm{cb}}=\sup _{n}\left\|\left(\gamma_{m+1, Z}\right)_{n}\right\| \leq\|u\|_{\mathrm{cb}}^{m+1}
$$

This establishes (b) for $m+1$ and completes the induction. The proof is complete on combining (9) and (14).

\section{Polynomials on Operator Hilbert Spaces}

In this section we discuss the operator space structure of the space of diagonal polynomials on the homogeneous operator Hilbert spaces $X$, where $X$ is $\mathrm{OH}$, $R$, or $C$. An operator space $X$ is homogeneous if $\|u\|=\|u\|_{\mathrm{cb}}$ for all $u \in$ $\mathrm{CB}(X, X)$. All closed subspaces of a homogeneous operator Hilbert space with the same Hilbert dimension are completely isometrically isomorphic (see [6] p. 172). We take a fixed orthonormal basis $\left(e_{j}\right)_{j=1}^{\infty}$ for $X$ with dual basis $\left(e_{j}^{*}\right)_{j=1}^{\infty}$. Let $\pi_{n}$ denote the projection from $X$ onto $X_{n}$, the span of $\left(e_{j}\right)_{j=1}^{n}$, $\pi^{n}=\mathbf{1}_{X}-\pi_{n}$, and let $Y$ denote an operator space. For any positive integer $m$ let

$$
\mathscr{P}_{\mathrm{cb}, d}\left({ }^{m} X, Y\right):=\mathscr{P}_{d}\left({ }^{m} X, Y\right) \cap \mathscr{P}_{\mathrm{cb}}\left({ }^{m} X, Y\right)
$$

where the diagonal polynomials are with respect to $\left(e_{j}\right)_{j=1}^{\infty}$. We endow $\mathscr{P}_{\mathrm{cb}, d}\left({ }^{m} X, Y\right)$ with its operator structure from $\mathscr{P}_{\mathrm{cb}}\left({ }^{m} X, Y\right)$. If $P \in \mathscr{P}_{\mathrm{cb}, d}\left({ }^{m} X, Y\right)$ then

$$
P\left(\sum_{j=1}^{\infty} x_{j} e_{j}\right)=\sum_{j=1}^{\infty} x_{j}^{m} P\left(e_{j}\right)=\left(\sum_{j=1}^{\infty} P\left(e_{j}\right)\left(e_{j}^{*}\right)^{m}\right)\left(\sum_{j=1}^{\infty} x_{j} e_{j}\right)
$$

and if $P_{n}:=P \circ \pi_{n}$ then, by (15),

$$
P_{n}=\sum_{j=1}^{n} P\left(e_{j}\right)\left(e_{j}^{*}\right)^{m}
$$

Proposition 4.1. If $m \geq 2$ and $Y$ is an operator space then $P \in$ $\mathscr{P}_{\mathrm{cb}, d}\left({ }^{m} \mathrm{OH}, Y\right)$ if and only if $\left(\left\|P\left(e_{j}\right)\right\|\right)_{n=1}^{\infty} \in c_{0}$. Moreover,

$$
\mathscr{P}_{d}\left({ }^{m} \mathrm{OH}, Y\right)=\mathscr{P}_{\mathrm{cb}, d}\left({ }^{m} \mathrm{OH}, Y\right),
$$

and

$$
\|P\|_{\bullet}=\|\bar{P}\|_{\mathrm{cb}}=\|P\|=\sup _{n}\left\|P\left(e_{j}\right)\right\| .
$$


Proof. If $P \in \mathscr{P}\left({ }^{m} \mathrm{OH}, Y\right)$ then $\left\|P\left(e_{j}\right)\right\| \leq\|P\|$ for all $j$. If $P \in$ $\mathscr{P}_{d}\left({ }^{m} \mathrm{OH}, Y\right)$ then $\left\|P\left(e_{j+1}\right)\right\| \leq\left\|P_{j+1}-P_{j}\right\| \rightarrow 0$ as $j \rightarrow \infty$ and $\left(\left\|P\left(e_{j}\right)\right\|\right)_{j=1}^{\infty}$ $\in c_{0}$.

Conversely, if $\left(\left\|y_{j}\right\|_{Y}\right)_{j=1}^{\infty} \in c_{0}$ let $P\left(\sum_{j=1}^{\infty} x_{j} e_{j}\right)=\sum_{j=1}^{\infty} x_{j}^{m} y_{j}$. Since

$$
\left\|\sum_{j=1}^{\infty} x_{j}^{m} y_{j}\right\| \leq \sup _{j}\left\|y_{j}\right\|_{Y} \cdot \sum_{j=1}^{\infty}\left|x_{j}\right|^{m} \leq \sup _{j}\left\|y_{j}\right\|_{Y} \cdot\left(\sum_{j=1}^{\infty}\left|x_{j}\right|^{2}\right)^{m / 2}<\infty,
$$

$P \in \mathscr{P}\left({ }^{m} \mathrm{OH}, Y\right)$ and $P\left(e_{j}\right)=y_{j}$ for all $j$. Moreover, as $n \rightarrow \infty$,

$$
\left\|P-P_{n}\right\| \leq \sup \left\{\left\|\sum_{j=n+1}^{\infty} x_{j}^{m} y_{j}\right\|:\left\|\sum_{j=1}^{\infty} x_{j}\right\| \leq 1\right\} \leq \sup \left\{\left\|y_{j}\right\|_{Y}: j \geq n\right\} \rightarrow 0
$$

and this implies $P \in \mathscr{P}_{d}\left({ }^{m} X, Y\right)$ and $\sup _{j}\left\|y_{j}\right\|_{Y}=\|P\|$.

Now fix a positive integer $n$ and let $P_{n}\left(\sum_{j=1}^{\infty} x_{j} e_{j}\right)=\sum_{j=1}^{n} x_{j}^{m+1} y_{j}$. As OH is self-dual and $\bar{\otimes}_{\min }$ is commutative, (4) and Proposition 3.4(a) implies

$$
\begin{aligned}
\left\|P_{n}\right\|_{\bullet} & =\left\|\sum_{j=1}^{n} e_{j}^{*} \otimes\left(e_{j}^{*} \otimes \cdots \otimes e_{j}^{*} \otimes y_{j}\right)\right\|_{\left(\bar{\otimes}_{\min }^{m+1} \mathrm{OH}^{*}\right) \bar{\otimes}_{\min } Y} \\
& =\| \sum_{j=1}^{n}\left(e_{j}^{*} \otimes \cdots \otimes e_{j}^{*} \otimes y_{j}\right) \\
& \otimes\left(e_{j}^{*} \otimes \cdots \otimes e_{j}^{*} \otimes \bar{y}_{j}\right) \|_{\left(\left(\bar{\otimes}_{\min }^{m} \mathrm{OH}^{*}\right) \bar{\otimes}_{\min } Y\right) \bar{\otimes}_{\min }\left(\left(\bar{\otimes}_{\min }^{m} \mathrm{OH}^{*}\right) \bar{\otimes}_{\min } Y\right)}^{1 / 2} \\
= & \left\|\sum_{j=1}^{n}\left(e_{j}^{*} \otimes \cdots \otimes e_{j}^{*}\right) \otimes y_{j} \otimes \bar{y}_{j}\right\|_{\left(\bar{\otimes}_{\min }^{2 m-1} \mathrm{OH}^{*}\right) \bar{\otimes}_{\min }\left(Y \bar{\otimes}_{\min } Y\right)}^{1 / 2} .
\end{aligned}
$$

By induction, we obtain for all $k$ a positive integer $\delta(k)$ such that

$$
\begin{aligned}
\left\|P_{n}\right\|_{\bullet}= & \| \sum_{j=1}^{n}\left(e_{j}^{*} \otimes \cdots \otimes e_{j}^{*}\right) \otimes\left(y_{j} \otimes \overline{y_{j}}\right) \otimes \\
& \cdots \otimes\left(y_{j} \otimes \overline{y_{j}}\right)\left\|_{\left(\otimes_{\min }^{\delta(k)} \mathrm{OH}^{*}\right) \bar{\otimes}_{\min }\left(\otimes_{\min }^{2^{k}} Y\right)}^{1 / 2^{k}} \leq\left(\sum_{j=1}^{n}\left\|y_{j}\right\|_{Y}^{2^{k}}\right)^{1 / 2^{k}} \rightarrow \sup _{j \leq n}\right\| y_{j} \|_{Y}
\end{aligned}
$$

as $k \rightarrow \infty$, since $\left\|y_{j}\right\|_{Y}=\left\|\overline{y_{j}}\right\|_{Y}$ for all $j$ and $\left\|e_{j}^{*} \otimes \cdots \otimes e_{j}^{*}\right\|_{\bigotimes_{\min }^{l} \mathrm{OH}^{*}}=1$ for any positive integer $l$. Hence $\left\|P_{n}\right\|_{\bullet}=\sup _{j \leq n}\left\|y_{j}\right\|_{Y}$ and for $s \geq t$

$$
\left\|P_{s}-P_{t}\right\| \leq\left\|P_{s}-P_{t}\right\|_{\bullet}=\sup _{t \leq j \leq s}\left\|y_{j}\right\|_{Y} \rightarrow 0 \quad \text { as } \quad s, t \rightarrow \infty .
$$


This implies $P \in \mathscr{P}_{\mathrm{cb}, d}\left({ }^{m} \mathrm{OH}, Y\right)$ and

$$
\|P\|_{\bullet}=\sup _{j}\left\|y_{j}\right\|_{Y} .
$$

Corollary 4.2. $\mathscr{P}_{\mathrm{cb}, d}\left({ }^{m} \mathrm{OH}\right)$ is a minimal operator space.

Proof. Let $\bar{N}:=N \cup(\infty)$ denote a one-point compactification of $N$ and let $\mathscr{C}_{0}(\bar{N})$ denote the set of all complex-valued continuous functions on $\bar{N}$ which vanish at infinity. With the supremum norm this is a commutative $\mathscr{C}^{*}$-algebra. Let $\phi: \mathscr{P}_{\mathrm{cb}, d}\left({ }^{m} \mathrm{OH}\right) \rightarrow \mathscr{C}_{0}(\bar{N})$ be defined as follows: if $\left(\lambda_{j}\right)_{j=1}^{\infty} \in c_{0}$ and

$$
P\left(\sum_{j=1}^{\infty} x_{j} e_{j}\right)=\sum_{j=1}^{\infty} \lambda_{j} x_{j}^{m}
$$

let

$$
[\phi(P)](j)=P\left(e_{j}\right)=\lambda_{j}, \quad[\phi(P)](\infty)=0 .
$$

Then

$$
\phi_{n}: M_{n}\left(\mathscr{P}_{\mathrm{cb}, d}\left({ }^{m} \mathrm{OH}\right)\right)=\mathscr{P}_{\mathrm{cb}, d}\left({ }^{m} \mathrm{OH}, M_{n}\right) \rightarrow \mathscr{C}_{0}\left(\bar{N}, M_{n}\right)=M_{n}\left(\mathscr{C}_{0}(\bar{N})\right)
$$

is given by $\left[\phi_{n}\left(\left[P_{i j}\right]_{1 \leq i, j \leq n}\right)\right](k)=\left[P_{i j}\left(e_{k}\right)\right]_{1 \leq i, j \leq n}=P\left(e_{k}\right)$ for all $k \in \bar{N}$ and all $P:=\left[P_{i j}\right]_{1 \leq i, j \leq n}$. By (19), $\left\|\phi_{n}\right\|=1$ for all $n$, that is $\phi$ is a complete isometry. By Proposition 3.3.1 in [2], $\mathscr{P}_{\mathrm{cb}, d}\left({ }^{m} \mathrm{OH}\right)$ is minimal. This completes the proof.

Proposition 4.3. If $R$ is the separable row operator Hilbert space and $R=$ $C^{*}$ then, for any positive integer $m, \mathscr{P}_{\mathrm{cb}, d}\left({ }^{m} R\right)=C$ completely isometrically.

Proof. We first suppose that $R:=R_{n}$ has finite dimension $n$. By (15) and (16) we see that $\mathscr{P}_{\mathrm{cb}, d}\left({ }^{m} R_{n}\right)$ is an $n$ dimensional space. We suppose, for fixed $m$, that $\mathscr{P}_{\mathrm{cb}, d}\left({ }^{m} R_{n}\right)$ is completely isometrically isomorphic to $\left(R_{n}\right)^{*}=C_{n}$. When $m=1$ the result is known from the linear theory. By definition the mapping

$$
\chi: P \in \mathscr{P}_{\mathrm{cb}, d}\left({ }^{m+1} R_{n}\right) \rightarrow \bar{P} \in \mathrm{CB}\left(X, \mathscr{P}_{\mathrm{cb}}\left({ }^{m} R_{n}\right)\right)=R_{n}^{*} \bar{\otimes}_{\min } \mathscr{P}_{\mathrm{cb}}\left({ }^{m} R_{n}\right)
$$

is a complete isometric embedding. Let $\lambda=\left(\lambda_{j}\right)_{j=1}^{n}$ and $x=\left(x_{j}\right)_{j=1}^{n}$ denote sequences of complex numbers. Let $P_{m, \lambda}=\sum_{j=1}^{n} \lambda_{j}\left(e_{j}^{*}\right)^{m} \in \mathscr{P}_{\mathrm{cb}, d}\left({ }^{m} X\right)$ and let $x=\sum_{j=1}^{n} x_{j} e_{j} \in R_{n}$. Since $\overline{P_{m+1, \lambda}}(x)=P_{m, \lambda x} \in \mathscr{P}_{\mathrm{cb}, d}\left({ }^{m} R_{n}\right)$ where $\lambda x=\left(\lambda_{j} x_{j}\right)_{j=1}^{n}$ for all $x \in R_{n}$ induction implies that $\chi\left(\mathscr{P}_{\mathrm{cb}, d}\left({ }^{m+1} R_{n}\right)\right) \subset R_{n}^{*} \bar{\otimes}_{\min } \mathscr{P}_{\mathrm{cb}, d}\left({ }^{m} R_{n}\right)=R_{n}^{*} \bar{\otimes}_{\min } R_{n}^{*}=C_{n} \bar{\otimes}_{\min } C_{n}=C_{n^{2}}$ 
and $\chi: \mathscr{P}_{\mathrm{cb}, d}\left({ }^{m+1} R_{n}\right) \rightarrow C_{n^{2}}$ is a complete isometric embedding. Since $C_{n^{2}}$ is homogeneous and contains $C_{n}$ as a subspace all $n$ dimensional subspaces of $C_{n^{2}}$ are completely isometric to $C_{n}$. Hence $\mathscr{P}_{\mathrm{cb}, d}\left({ }^{m+1} R_{n}\right)=C_{n}$ completely isometrically. By induction this completes the proof when $R$ is finite dimensional.

When $R$ is infinite dimensional the above proof can be adapted to show that $\mathscr{P}_{\mathrm{cb}, d}\left({ }^{m} R\right)$ is completely isometric to the closure of an infinite dimensional subspace of $R^{*} \otimes R^{*}$ in $\mathrm{CB}\left(R, R^{*}\right)$. Hence $\mathscr{P}_{\mathrm{cb}, d}\left({ }^{m} R\right)$ is completely isometrically isomorphic to an infinite dimensional Banach subspace of $R^{*} \bar{\otimes}_{\min } R^{*}=R^{*}=$ $C$. By homogeneity this implies $\mathscr{P}_{\mathrm{cb}, d}\left({ }^{m} R\right)=C$ completely isometrically and completes the proof.

We remark that a similar proof shows that $\mathscr{P}_{\mathrm{cb}, d}\left({ }^{m} C\right)=R$ completely isometrically for all $m$ and that we can also obtain the same result for nonseparable spaces.

Both authors are very grateful to the referee for comments which led to the removal of a number of errors and to clarifications in the presentation.

\section{REFERENCES}

1. Dineen, S., Complex Analysis on Infinite-Dimensional Spaces, Springer, London 1999.

2. Effros, E. G., Ruan, Z-J., Operator Spaces, London Math. Soc. Monographs 23, Oxford Univ. Press, Oxford 2000.

3. Harris, L. A., Bounds on the derivatives of holomorphic functions, pp. 145-163 in: Analyse fonctionnelle et applications, Proc. Rio de Janeiro 1972, Actualités Sci. Indust. 1367, Herman, Paris 1975.

4. Murphy, G. J., $C^{*}$-Algebras and Operator Theory, Academic Press, Boston 1990.

5. Paulsen, V., Completely Bounded Maps and Operator Algebras, Cambridge Studies in Adv. Math. 78, Cambridge Univ. Press, Cambridge 2002.

6. Pisier, G., Introduction to Operator Space Theory, London Math. Soc. Lecture Note 294, Cambridge Univ. Press, Cambridge 2003.

7. Pisier, G., The Operator Space OH, Complex Interpolation and Tensor Norms, Mem. Amer. Math. Soc. 122:585 (1996).

8. Pisier, G., Completely bounded maps into certain Hilbertian operator spaces, Int. Math. Res. Not. 2004:74, 3983-4018.

9. Pisier, G., Non-Commutative vector valued $L_{p}$-spaces and completely p-summing maps, Asterisque 247 (1998).

10. Pisier, G., Complex interpolation and regular operators between Banach lattices, Archiv Math. (Basel) 62 (1994), 261-269.

11. Ruan, Z-J., Subspaces of $C^{*}$-algebras, J. Funct. Anal. 76 (1988), 217-230.

SCHOOL OF MATHEMATICAL SCIENCES

UNIVERSITY COLLEGE DUBLIN

BELFIELD

DUBLIN 4

IRELAND

E-mail: sean.dineen@ucd.ie cristina.radu@ucd.ie 